\title{
Dynamics of protein-protein encounter: a Langevin equation approach with reaction patches
}

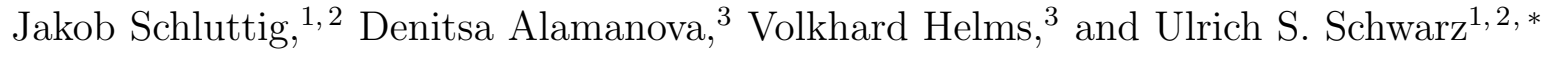 \\ ${ }^{1}$ University of Heidelberg, Bioquant 0013, \\ Im Neuenheimer Feld 267, 69120 Heidelberg, Germany \\ ${ }^{2}$ University of Karlsruhe, Theoretical Biophysics Group, \\ Kaiserstrasse 12, 76131 Karlsruhe, Germany \\ ${ }^{3}$ Center for Bioinformatics, Saarland University, 66041 Saarbrücken, Germany
}

(Dated: July 6, 2021) 


\begin{abstract}
We study the formation of protein-protein encounter complexes with a Langevin equation approach that considers direct, steric and thermal forces. As three model systems with distinctly different properties we consider the pairs barnase:barstar, cytochrome c:cytochrome c peroxidase and p53:MDM2. In each case, proteins are modeled either as spherical particles, as dipolar spheres or as collection of several small beads with one dipole. Spherical reaction patches are placed on the model proteins according to the known experimental structures of the protein complexes. In the computer simulations, concentration is varied by changing box size. Encounter is defined as overlap of the reaction patches and the corresponding first passage times are recorded together with the number of unsuccessful contacts before encounter. We find that encounter frequency scales linearly with protein concentration, thus proving that our microscopic model results in a well-defined macroscopic encounter rate. The number of unsuccessful contacts before encounter decreases with increasing encounter rate and ranges from 20-9000. For all three models, encounter rates are obtained within one order of magnitude of the experimentally measured association rates. Electrostatic steering enhances association up to 50-fold. If diffusional encounter is dominant (p53:MDM2) or similarly important as electrostatic steering (barnase:barstar), then encounter rate decreases with decreasing patch radius. More detailed modeling of protein shapes decreases encounter rates by 5-95 percent. Our study shows how generic principles of protein-protein association are modulated by molecular features of the systems under consideration. Moreover it allows us to assess different coarse-graining strategies for the future modelling of the dynamics of large protein complexes.
\end{abstract}

*E-mail: ulrich.schwarz@bioquant.uni-heidelberg.de 


\section{INTRODUCTION}

Protein-protein interactions play key roles in many cellular processes such as signal transduction, bioenergetics, and the immune response [1]. Moreover, many proteins function in the context of protein complexes of variable sizes and lifetimes. Examples of such complexes are ribosomes, polymerases, spliceosomes, nuclear pore complexes, cytoskeletal structures like the mitotic spindle or actin stress fibers, adhesion contacts, the anaphase-promoting complex, and the endocytotic complex [2]. For yeast, 800 different core complexes have been identified, suggesting the existence of 3000 core complexes for humans [3]. In addition it has been shown for yeast that most protein complexes are assembled just-in-time during the course of the cell cycle [4]. In fact many protein complexes in the cell are highly dynamic, with fast turnover of many components. One can argue that their dynamics, although experimentally very hard to access, is biologically more relevant than their equilibrium properties. Therefore a systematic understanding of the dynamics of protein complexes in cells is one of the grand challenges in quantitative biology.

The elementary unit of all of these cellular processes is the bimolecular protein-protein interaction. The strength and specificity of protein-protein association are determined by the integrated effect of different interactions, including shape complementarity, van der

Waals interactions, hydrogen bonding, electrostatic interactions and hydrophobic effects. For example, the importance of electrostatic interactions has been demonstrated by experimental measurement at different ionic strengths [5]. To a first approximation, bimolecular reactions are characterized by on- and off-rates. The equilibrium association constant (or affinity) then follows as the ratio of the two. From a conceptual point of view, on- and off-rates are very different. On-rates are commonly believed to be controlled by the diffusion properties as well as by long-ranged electrostatic interactions, whereas off-rates are rather controlled by short-ranged interactions like hydrogen bonding and van der Waals forces.

The main features of the dynamics of protein association can be conceptualized within the framework of the encounter complex [6]. To this end, the association is divided into two parts. First, mutual entanglement - the encounter complex - is achieved by the proteins due to a transport process including mainly diffusion but also electrostatic steering on small length scales [7]. If diffusion-controlled, classical continuum approaches can be used to describe this part of the process [8]. To form the final complex, the system then has to 
overcome a free energy barrier due to local effects like dehydration of the binding interface [9]. Due to the various molecular contributions involved in this step, here the two binding partners essentially have to be modelled at atomic detail. Moreover the solvent may need to be treated explicitly and one might has to account for conformational changes [10]. Thus, it appears reasonable to use the encounter complex as a crossover point from a detailed, atomistic treatment to a coarse-grained model and vice versa.

Thermal fluctuations are an essential element of protein-protein encounter because they allow the two partners to exhaustively search space for access to the binding interface. From the viewpoint of stochastic dynamics, protein-protein association is a first passage time problem which can be addressed mathematically in the framework of Langevin equations. The application of Langevin equations to association phenomena goes back to early work in the colloidal sciences [11, 12]. In these early approaches, the reactants were considered to have small spatial extensions and to be uniformly reactive. For large biomolecules like proteins, the situation is fundamentally different. Typically, proteins and other biomolecules have specific sites on their surface, where a particular binding reaction can take place. Therefore, such binding events are subject to intrinsic geometric constraints for every particular protein-protein pair or larger assembly. The standard model for ligand-receptor interaction was introduced by Berg and Purcell in the context of chemoreception based on the idea of using reactive patches to model anisotropic reactivity [13]. Due to anisotropic reactivity, also rotational diffusion becomes important. Shoup et al. showed that the effect of rotational diffusion can strongly increase the association rate between a receptor with a flat reactive patch and uniformly reactive ligands [14]. Later, analytic expressions for the association rate between two spherical particles with both carrying a flat axially symmetric [15] and asymmetric [16, 17] reactive patch were derived. Similar concepts were also applied by Schulten and colleagues [18].

For many important aspects, analytical approaches are not possible and computer simulations are required. This approach has been used early for protein-protein association [19]. The importance of electrostatic interactions for long ranged attraction was also emphasized by Brownian dynamics simulations of protein-protein encounter [19, 20, 21, 22]. If atomic structure is taken into account, then successful encounters are defined by simultaneous fulfillment of two to three distance conditions between opposing residues on the two surfaces [23. Brownian dynamics have also been used for the simulation of high density solutions, 
e.g. by Bicout and Field who studied a cellular "soup" containing ribosomes, proteins and tRNA molecules [24], or by Elcock and coworkers who simulated a crowded cytosol for $10 \mu \mathrm{s}$ [25].

In order to develop a quantitative framework for modelling the dynamics of protein complexes, it is essential to understand the relative importance of generic principles and molecularly determined features of specific systems of interest. Only a good understanding of this issues will allow us in the future to develop reasonable coarse-graining strategies to address also large complexes of biological relevance. In this study, we therefore address how general principles guiding the diffusional association of biomolecular pairs are modulated by their particular physicochemical properties. To this end we have selected three molecular systems of interest with different steric and electrostatic properties. One of the best studied bimolecular complexes is the extracellular ribonuclease Barnase and its intracellular inhibitor Barstar. Both proteins carry a net charge of $2 e$ and $-6 e$, respectively, which leads to a considerable electrostatic steering [26, 27, 28, 29, 30]. Considering the structure of the two proteins, Barnase has a bean-like form, matching well on a large reactive area with the nearly spherical Barstar. A classic example of electrostatically-driven protein association is the iso-1-cytochrome $c$ - cytochrome c peroxidase (Cytc:CCP) complex, charged with $6 e$ and $-13 e$, respectively, and exhibiting dipoles aligned well with the reactive areas [19, 31]. Finally, we selected the medically important complex of a peptide fragment of p53 and its inhibitor MDM2, which is used for anticancer drug design. In this system, electrostatic attraction plays a minor role. On the other hand, the steric match of the two surfaces is of particular importance here. It is a perfect example of a key-lock binding interface, where p53 is buried deep into a cleft on the MDM2 surface.

In this paper we systematically explore the effect of various coarse-graining procedures on the rate for protein-protein encounter for the three selected model systems. We revisit early approaches based on Langevin equations and combine them with current knowledge on molecular structure. The paper is organized as follows: In Sect. [II] we present our different stochastic models and describe the methods we use to parameterize the three considered bimolecular model systems. Sect. III contains the main findings of our study, which are discussed and summarized in Sect. [V] 


\section{MODELS AND METHODS}

\section{A. Modelling proteins at different levels of detail}

One aim of this work is to determine how important specific details of the model proteins are with respect to the association properties. Therefore, we considered three different levels of detail as depicted in Fig. 1 for the three chosen systems. In the most generic approach $(\mathcal{M} 1)$, we only considered the steric interaction between spherical particles covered with reaction patches. As a first refinement $(\mathcal{M} 2)$, an effective Coulombic interaction was introduced using the dipolar sphere model (DSM). Finally, since our Langevin equation approach is particularly suited to capture anisotropic transport, we consider a more refined version for protein sterics $(\mathcal{M} 3)$. In this approach the excluded volume of each protein was modeled by 8-25 smaller beads. $\mathcal{M} 3$ uses the DSM as well. In Fig. 1, we also show the full structures in the bottom row as surface representations, including the locations of the binding interfaces. In the following, the general properties of the simulation model and the different techniques used in this work will be explained.

\section{B. Diffusion properties}

The diffusion of the protein model particles is described by an anisotropic $6 \times 6$ diffusion matrix in all versions of our model. In Ref. [32], de la Torre and coworkers present a method to calculate this diffusion matrix from the pdb structure of a protein. This method has been implemented in a software called HYDROPRO which is provided online by the same authors (http://leonardo.fcu.um.es/macromol). The basic concept is to put spheres of a certain size at the position of any non-H atom. The volume of these spheres effectively models a fixed hydration shell. This construct is then filled up with smaller, densely packed, but nonoverlapping spheres. Since the hydrodynamic properties of a rigid body are determined by its outer boundary only, a shell of these small spheres is generated by deleting all spheres which have a maximum number of possible neighbors. To this shell a sophisticated technique is applied, which has been developed by de la Torre and colleagues over the years, to calculate the diffusion matrix of such a cluster of non-overlapping spheres (see references in [32]). Several system properties are implicitely contained in the mobility matrix, such as ambient temperature $T_{a}=293 \mathrm{~K}$, as well as the density and dynamic viscosity of the solvent, where 
we chose the respective parameters of water, $\rho=1 \mathrm{~g} / \mathrm{cm}^{3}$ and $\mu=10^{-3} \mathrm{~Pa}$ s. For simplicity, hydrodynamic interactions were not introduced in our models, because the corresponding effect on the association rates is expected to be well below $10 \%$ [33].

\section{Langevin equation and simulation method}

For the integration of the Langevin equation, which describes the stochastic motion of the particles, we follow an approach which has been recently developed to model cell adhesion via reactive receptor patches [34, 35]. Let $\mathbf{X}_{t}$ be a six-dimensional vector describing position and orientation of a particle at time $t$. Since the noise due to Brownian motion is additive (which means that it does not depend on $\mathbf{X}_{t}$ due to a constant mobility matrix $\mathbf{M}$ ), the Langevin equation is given by:

$$
\partial_{t} \mathbf{X}_{t}=\mathbf{M F}+\mathbf{g}_{t}
$$

Here, $\mathbf{F}$ is a six-dimensional vector containing the force and torque acting on the particle, and $\mathbf{g}_{t}$ denotes Gaussian white noise:

$$
\left\langle\mathbf{g}_{t}\right\rangle=0, \quad\left\langle\mathbf{g}_{t} \mathbf{g}_{t^{\prime}}\right\rangle=2 k_{B} T_{a} \mathrm{M} \delta\left(t-t^{\prime}\right) .
$$

As explained in App. C of Ref. [35], the Euler algorithm can be used to solve a discretized version of this equation:

$$
\mathbf{X}(t+\Delta t)=\mathbf{X}(t)+\mathbf{M F}(t) \Delta t+\mathbf{g}(\Delta t)+\mathcal{O}\left(\Delta t^{2}\right)
$$

For proteins, the typical orders of magnitude are $D=10^{-6} \mathrm{~cm}^{2} \mathrm{~s}^{-1}$ and $R=1 \mathrm{~nm}$. Therefore, a reasonable choice for the time step is $\Delta t=1 \mathrm{ps}$, as this leads to a mean step length of $\sqrt{D \Delta t}=0.01 \mathrm{~nm}$.

The mobility matrix of a particle is defined in a particle-fixed coordinate system. Thus, the whole step has to be calculated in terms of particle-fixed coordinates and then transformed to the laboratory coordinate space. In particular, this transformation implies a rotation $\mathrm{R}$ regarding the orientation of the particle. Special attention has to be payed to the force $\mathbf{F}$, which is typically calculated in the global frame of reference and hence has to be transformed to particle space before Eq. 3 can be evaluated. This back-transformation 
is achieved by applying $R^{-1}$ to $\mathbf{F}$. Since rotation matrices $\mathrm{R}$ simply consist of a list of orthonormal vectors, their inverse is equal to the transposed matrix $\mathrm{R}^{-1}=\mathrm{R}^{T}$. Thus Eq. 3 can be rewritten as

$$
\mathbf{X}(t+\Delta t)=\mathbf{X}(t)+\mathrm{R}\left[\mathrm{M}\left(\mathrm{R}^{T} \mathbf{F}(t)\right) \Delta t+\mathbf{g}(\Delta t)\right]+\mathcal{O}\left(\Delta t^{2}\right)
$$

As $\mathbf{F}$ and $\mathbf{g}$ are six-dimensional and contain information about torque and rotation, Eq. 4 is only formally correct, as R acts on both the translational and rotational parts of the respective vectors separately.

In each step of the simulation, a displacement vector $\Delta \mathbf{X}(t)$ is drawn for each particle as described above. If this global displacement leads to any violation of the hardcore repulsion, all suggested displacements are rejected and new $\Delta \mathbf{X}(t)$ are calculated. This procedure continues, until an update of all positions and orientations is found which does not lead to any overlap. In this way, the constraint according to the excluded volume effect is included in the stochastic motion. The spherical reactive patches are not taken into account for the steric interactions, i.e. they may not only overlap pairwise but also with the model particles. One would expect that our procedure leads to errors of order $\Delta t$ if two particles are in close proximity of order $\sqrt{D \Delta t}$. However, it has been shown for a different system [36] that in practise the deviation from the expected behavior is very small and thus the approach is reasonable.

\section{Anisotropic versus isotropic diffusion}

As mentioned before, the $6 \times 6$ mobility matrix $M$ represents anisotropic diffusion. For large times, anisotropic diffusion crosses over into isotropic diffusion because the information about the initial orientation gets lost after a certain relaxation time due to the rotational diffusion [37]. In general, translational and rotational diffusion are coupled so that large time steps cannot be used. However, for the particular systems studied here, we found that the diffusive coupling is a very small effect. In particular, the major entries in the diffusion matrix of the proteins used here according to HYDROPRO multiplied with different powers of the Stokes radius $R \sim 10^{-7} \mathrm{~cm}$ to make the dimensions comparable are $D_{\mathrm{tt}} / R^{2} \sim 10^{8} \mathrm{~s}^{-1}$, $D_{\mathrm{rr}} \sim 10^{7} \mathrm{~s}^{-1}, D_{\mathrm{tr}} / R \sim 10^{5} \mathrm{~s}^{-1}$. Therefore, the effect of diffusive coupling is $10^{-2}$ and $10^{-3}$ smaller than rotational and translational diffusion, respectively. Finally, the typical time 
scale at which the cross-over is expected can be calculated to be $1 /\left(6 D_{\mathrm{rr}}\right) \approx 10 \mathrm{~ns}$. Time steps of this magnitude were rarely used in the simulations (see below), so that for most of the steps, the anisotropicity is well preserved. Therefore we can safely neglect changes in the anisotropicity of the mobility matrix.

\section{E. System size and time step adaption}

The simulations were performed in a cubic box with periodic boundary conditions. Schreiber and Fersht used concentrations between $0.125 \mu \mathrm{M}$ and $0.5 \mu \mathrm{M}$ in their experimental studies of the association rate of the Barnase:Barstar complex [5]. The average volume containing one particle at a concentration $c$ is $1 / c N_{A}$ with the Avogadro number $N_{A}=6 \cdot 10^{23} \mathrm{~mol}^{-1}$. Hence, the edge length of a cubic boundary box representing concentration $c$ can be calculated from $L=\sqrt[3]{V}=1 / \sqrt[3]{c N_{A}}$. E.g. $c=0.125 \mu$ M leads to $L \approx 2370 \AA$ for one pair of particles, which is two orders of magnitude larger than the size of the proteins. Due to this low density, the first passage times (FPT) for encounter can be expected to be much longer than the chosen time step. For computational efficiency, we therefore used a variable time step in our simulations. Van Zon and ten Wolde suggested a method to avoid unwanted collisions when they introduced their Green's function reaction dynamics (GFRD) [38]. In contrast to our work, however, this method is based on isotropic diffusion. Generalizing the GFRD to anisotropic diffusion is out of the scope of our work and we therefore used the following scheme. We first note that in GFRD each time step is chosen such that it includes the next reaction. In our case, we also want to investigate the stochastic dynamics before the next encounter event takes place. Thus a large time step is not chosen to include the next encounter, but to bring the system to such a configuration that encounter becomes more likely. This step can be well represented by isotropic diffusion with an overall diffusion constant $D=\left(\mathcal{D}_{11}+\mathcal{D}_{22}+\mathcal{D}_{33}\right) / 3$ following from the anisotropic diffusion matrix.

For an isotropic random walk, the displacement probability is given by a Gaussian distribution with spherical symmetry. Thus, large spatial steps are exponentially suppressed, which makes a step of size $\Delta r_{\max }^{H} \geq H \sqrt{6 D \Delta t}$ an $H$-sigma event. By setting $\Delta r_{\max }^{H}=\min \left\{r_{i j}^{\text {eff }}\right\}$ the smallest effective particle distance in the system, where effective means the distance of the surfaces $r_{i j}^{\mathrm{eff}}=\left|\mathbf{r}_{i}-\mathbf{r}_{j}\right|-R_{i}-R_{j}$ with $R_{i}$ determining the maximal steric interaction radius of particle $i$, one can estimate a reasonable time step for which a 
collision is highly improbable. Van Zon and ten Wolde found that the choice $H=3$ provides good results, combined with the fact that wrongly sampling a collision event would need a certain direction of the displacement in addition to the length. As the particles reach close

proximity $\min \left\{r_{i j}^{\text {eff }}\right\} \rightarrow 0$, the estimated $\Delta t$ vanishes and thus the simulation would be slowed down infinitely. Therefore, there has to be some lower boundary for the time step $\Delta t_{\min }$, which is generally chosen to be $\Delta t_{\text {min }}=1$ ps as explained earlier. Thus, the adapted time step is given by:

$$
\Delta t_{\mathrm{ad}}=\min \left\{\frac{H^{2}}{6 D}\left(\min \left\{r_{i j}^{\mathrm{eff}}\right\}\right)^{2} ; \Delta t_{\mathrm{min}}\right\} .
$$

In practice, most time steps are in the ps-range, with very few time steps coming up to the ns-range.

\section{F. Electrostatic interactions}

Electrostatic interactions are known to play an important role in protein association. To study the effect of electrostatics in our generic model, the models $\mathcal{M} 2$ and $\mathcal{M} 3$ utilized the dipolar sphere model (DSM), following Refs. [39, 40]. The DSM effectively models a monopole and dipole interaction by summing over the interactions of three charges, one positioned in the center of each particle, and two close to its surface in opposite positions. Taking into account the Debye screening function due to the presence of counter ions in solution, the electrostatic interaction energy between two charges $q_{i / j}$ at positions $\mathbf{r}_{i / j}$ respectively with distance $r_{i j}=\left|\mathbf{r}_{i j}\right|=\left|\mathbf{r}_{j}-\mathbf{r}_{i}\right|$ is:

$$
W_{i j}=\frac{1}{4 \pi \varepsilon_{0} \varepsilon_{r}} q_{i} q_{j} \frac{e^{-\kappa\left(r_{i j}-B_{i j}\right)}}{\left(1+\kappa B_{i j}\right) r_{i j}} .
$$

Here, $\kappa=l_{D}^{-1}$ is the inverse Debye screening length, which typically has a value of $\approx$ $1 \mathrm{~nm}$ under physiological conditions. We assume a value of $\varepsilon_{r}=78$ for the relative static permittivity of the medium, which reflects the properties of water at ambient temperature. $B_{i j}$ is a correction to the screening of charges which are placed in an object like a protein which has no free charges inside. Taking $b_{i / j}$ as the closest distance of $q_{i / j}$ from the surface of the surrounding protein, it is approximately given by $B_{i j}=b_{i}+b_{j}$. This potential leads 
to a force of charge $q_{j}$ on $q_{i}$ :

$$
\begin{aligned}
\mathbf{F}_{i j} & =-\nabla_{\mathbf{r}_{i}} W_{i j}=-\frac{\partial W_{i j}}{\partial r_{i j}} \cdot\left(\nabla_{\mathbf{r}_{i}} r_{i j}\right) \\
& =-\frac{1}{4 \pi \varepsilon_{0} \varepsilon_{r}} q_{i} q_{j} \frac{e^{-\kappa\left(r_{i j}-B_{i j}\right)}\left(1+\kappa r_{i j}\right)}{\left(1+\kappa B_{i j}\right) r_{i j}^{2}} \frac{\mathbf{r}_{i j}}{r_{i j}} .
\end{aligned}
$$

As our simulation uses periodic boundary conditions, actually an infinite number of copies exists for every charge. However, due to the very fast decay of the screened electrostatic interaction, only the minimum image distance of two charges is considered in the force calculation. Two model particles $m$ and $n$ feel the sum of the Coulomb forces $\mathbf{F}_{i j}$ between all pairs of the three complementary charges mimicking the monopolar and dipolar interactions.

Thus the full force between particle $m$ and $n$ is $\mathbf{F}_{m n}=\sum_{i=1}^{3} \sum_{j=1}^{3} \mathbf{F}_{i j}$, where $i / j$ run over the charges of $m / n$ respectively.

As explained earlier, the action of the force on a particle in the Langevin equation is weighted with the mobility matrix M. The HYDROPRO software directly gives the diffusion matrix $\mathcal{D}=k_{B} T_{a} \mathrm{M}$. This means that in our case the force action should be rewritten as $\mathrm{MF} \Delta t=\mathcal{D} \mathbf{F} \Delta t / k_{B} T_{a}$. Considering a time step of $\Delta t=10 \mathrm{ps}$ and a typical diffusion constant $D=10^{-10} \mathrm{~m}^{2} / \mathrm{s}$, we have $D|\mathbf{F}(1 \mathrm{~nm})| \Delta t / k_{B} T_{a} \sim 10^{-13} \mathrm{~m}$ and $D|\mathbf{F}(4 \mathrm{~nm})| \Delta t / k_{B} T_{a} \sim 10^{-15} \mathrm{~m}$ for typical distances $r_{i j}=1 \mathrm{~nm}$ and $r_{i j}=4 \mathrm{~nm}$, respectively. In contrast, the typical step length due to the Brownian motion is $\sqrt{D \Delta t} \sim 10^{-11} \mathrm{~m}$. This shows that the magnitude of electrostatic interactions at distances of $1 \mathrm{~nm}$ is much smaller than thermal energy. Therefore the effect of force is also not considered in our adaptive scheme for the time steps. However, it can be expected that the systematic drift, albeit small, will still lead to an altered encounter behavior.

\section{G. Parameterization}

Gabdoulline and Wade [23] used several criteria to define contact areas of bimolecular protein complexes. In our studies, we define the contact area to consist of those atoms in the two interacting proteins that are at $5 \AA$ or less distance from an atom of the complementary protein. The center of mass of these atoms is considered as the center of the reactive area. For $\mathcal{M} 1$ and $\mathcal{M} 2$, the reactive patch is centered at the surface of the sphere modeling the excluded volume such that it has the same relative direction from the center of mass as 
obtained by the method described. In the case of $\mathcal{M} 3$, the center of the patch is set to the center of the reactive area.

The contact area has a diameter of approximately $10 \AA$ to $20 \AA$ for the three systems studied here. Following earlier Brownian dynamics simulations with atomistic details [7] we have performed an in-depth analysis of the free energy landscape and the encounter state of the protein complexes considered in this work (unpublished results). This showed that the encounter complex is typically located at relative separations of the two protein surfaces of about $10 \AA$ compared to their positions in the final complex. As the spherical reactive patches used in this study simultaneously determine both the size of the contact area on the surface and the distance above their surface at which an encounter will be possible, values in the range of $5 \AA$ to $10 \AA$ seem to be reasonable. Note that as long as physical considerations do not dictate non-spherical reaction patches, the spherical choice is highly favorable for computational efficiency.

As already stated in the beginning, two types of excluded volume structures are taken into account. In the first case, used in $\mathcal{M} 1$ and $\mathcal{M} 2$, the proteins are assumed to have an approximately spherical form. The radius for the model spheres determining the hard core interaction follows as the radius of gyration of the protein, which is also calculated by the HYDROPRO software. The underlying data in the more detailed approach $\mathcal{M} 3$ is obtained using the AtoB bead modeling software [41, 42]. In this way, the three-dimensional structure of the proteins is modeled with a comparably small number of 8 to 25 spheres of different sizes.

The monopole charge is the sum of all elementary charges in a protein and is placed at the center of the respective model particle. The dipole moment $\mathbf{p}$ is obtained by summing over the product of all atomic charges due to the xyz force field and their relative position to the center of mass. In the model, it is represented by two opposing charges which are positioned along the direction of $\mathbf{p}$ and at a distance $r_{p}=R_{\mathrm{gyr}}-4 \AA$. The magnitude $p^{\prime}$ is chosen such that $|\mathbf{p}|=2 p^{\prime} r_{p}$. We found that the particular choice of $r_{p}$ does not have a noticeable influence on the results. The resulting parameterization is given in Tab. If for the proteins considered here. 


\section{RESULTS}

\section{A. Encounter frequency and encounter rate}

Langevin dynamics simulations were performed for cubic boxes containing two model proteins. Simulations were conducted until the encounter condition was met for the first time (typically after milliseconds). Because in our Langevin simulations we measure the distribution of first passage times (FPT) to encounter, from which we can deduce the mean first passage time (MFPT) $\langle T\rangle$, the encounter frequency is defined as $k=1 /\langle T\rangle$. This choice is motivated by the fact that for a Poisson-like process, the distribution of first passage times is given by $f(T)=k e^{-k T}$ and therefore the encounter frequency $k$ indeed satisfies $k=1 /\langle T\rangle$. As the preparation of a comparable experiment would never allow knowing the particular initial positions and orientations of the unbound proteins, it makes sense to average over the possible initial configurations in the computer simulations. Therefore, we started a large number of runs (typically $10^{4}$ to $10^{5}$ ) with random initial positions and orientations for all involved model particles, under the constraint that the initial pairwise distance is at least large enough to prevent an immediate encounter. The "first passage" is defined as the first overlap of two complementary reactive patches. Interestingly, due to this averaging the first passage process becomes Poisson-like, see Fig. 2. The data show a clear exponential behavior. This means that it is justified to use the notion of an "encounter frequency", as the FPT distribution is indeed represented by a single stochastic rate. The finite probability at small FPT is due to the possibility that the two model particles are already in close proximity when the simulation is started. The large errors in the histogram at $T \rightarrow 0$ are caused by the fact that exponentially sized histogram bins were used to sample the behavior for small $T$. Therefore, events hitting a particular bin are rare because of the small width of the bins at $T \rightarrow 0$, which then leads to bad statistics in this domain.

As the encounter process is purely diffusion limited in $\mathcal{M} 1$, one would expect the encounter frequency to scale linearly with concentration. Fig. 3 demonstrates for the Barnase:Barstar system that this is indeed the case. Hence, it is reasonable to always scale the encounter frequencies with the inverse concentration, as will be done for the rest of this work. We will denote these rescaled quantities as encounter rate, i.e. the encounter rates have the dimension $\mathrm{M}^{-1} \mathrm{~s}^{-1}$. In summary, we have demonstrated here that our microscopic 
model leads to a well-defined macroscopic encounter rate.

\section{B. Finite size effects}

In most of the simulations, only one instance of the final complex was considered, i.e. one model particle of each kind. Using such small systems could lead to undesired finite size effects. We therefore considered the effect of having many particles in the simulation box. Fig. 4 shows the simulation results for the encounter frequency $k$ for an increasing number of Barnase:Barstar pairs, while keeping the size of the boundary box constant. In order to understand the expected effects, consider a system with molecules of Barnase $\left(A\right.$ and $\left.A^{\prime}\right)$ and two molecules of Barstar $\left(B\right.$ and $\left.B^{\prime}\right)$ randomly distributed over the boundary box. The relative alignment of any pair of $\mathrm{As}$ and $\mathrm{Bs}$ is therefore random again. For a particular pair the distribution of times to first encounter will thus look very similar to the case with a single pair in the box, which is a simple exponential decay with respect to the encounter frequency $k_{1}: f_{1}(T)=k_{1} \exp \left[-k_{1} T\right]$. The probability that, e.g., the particular pair $\mathrm{A}-\mathrm{B}$ reaches encounter at a certain time $t$ before the three other possible pairs $\left(A^{\prime}-B, A-B^{\prime}\right.$, $\left.\mathrm{A}^{\prime}-\mathrm{B}^{\prime}\right)$, is therefore:

$$
p(t)=\int_{0}^{\infty} \mathrm{d} t_{1} \int_{t_{1}}^{\infty} \mathrm{d} t_{2} \int_{t_{1}}^{\infty} \mathrm{d} t_{3} \int_{t_{1}}^{\infty} \mathrm{d} t_{4} \delta\left(t_{1}-t\right) \prod_{i=1}^{4} k_{1} e^{-k_{1} t_{i}}=k_{1} e^{-4 k_{1} t} .
$$

Thus, the probability that any of the four possible particle pairs reaches encounter before the respective three other pairs do, is $4 \times p(t)$ as just calculated, i.e. $f_{2}(T)$ has again a Poisson form like $f_{1}(T)$ and $k_{2}=4 k_{1}$. In general, for higher numbers of particle pairs $N$, we expect to again find an exponential distribution of the time to first encounter with the encounter frequency $k_{N}=N^{2} k_{1}$. This quadratic behavior is nicely confirmed by the data shown in Fig. 4, which suggests that even for small systems with only two particles, no severe finite size effects have to be expected. In particular, this rules out that larger numbers of particles lead to noticeable three-body interactions or hindering of the encounter process.

\section{Alignment during encounter}

One feature of special interest which we can address with our Langevin equation approach is the pathway through which the encounter is formed. We dissected the encounter process 
into several parts as visualized in Fig. 5. At the start of each run, the systems were prepared in the unaligned state $\mathcal{A} 1$, as described earlier. A state of close approach which however does not allow for binding is called $\mathcal{A} 2$. The two model proteins will switch between states $\mathcal{A} 1$ and $\mathcal{A} 2$ a number of times $N$, until they finally reach the encounter complex $\mathcal{A} 3$ due to a favorable combination of translational and rotational diffusion. In the following, each occurance of $\mathcal{A} 2$ will be termed a contact. Thus $N$ counts the number of unsuccessful contacts before the encounter is finally formed. A separate set of simulations was performed to measure the distribution of $N$. Furthermore we analyzed the distribution of return times $T_{\text {off. }}$ This is the time it takes for two model proteins to get into contact again $(\mathcal{A} 1 \rightarrow \mathcal{A} 2)$ after having lost translational alignment $(\mathcal{A} 2 \rightarrow \mathcal{A} 1)$, i.e. after they were in close proximity. Finally, we determined the distribution of resting times $T_{\text {on }}$ in translational alignment $\mathcal{A} 2$ before the two model particles separated again.

As an example, Fig. 6 shows the distribution of $N$ for the Barnase:Barstar model system at $c=0.5 \mu \mathrm{M}$ in the framework of $\mathcal{M} 1$. Surprisingly the distribution of the number of contacts has again a Poisson form. Note that the number of unsuccessful trials in state $\mathcal{A} 2$ can be rather large (up to $10^{4}$ ). We also found that the distribution of $N$ is roughly independent of concentration. This is reasonable, as after the two proteins were in contact once, the further encounter process is guided by returns to state $\mathcal{A} 2$ and thus should be more or less independent of system size.

Fig. 7 shows that the return time $T_{\text {off }}$ (plotted with the plus-symbol) is not exponentially distributed. Instead, it follows a power law $p\left(T_{\text {off }}\right) \sim T_{\text {off }}^{-3 / 2}$ and undergoes an exponential cutoff due to the finite size of the boundary box at large $T_{\text {off }}$. Therefore, there is a high probability for very small return times, i.e. situations, where the two model proteins do not really separate, but immediately after loosing translational alignment $(\mathcal{A} 2 \rightarrow \mathcal{A} 1)$ get closer again $(\mathcal{A} 1 \rightarrow \mathcal{A} 2)$. The power law behavior of the return time is consistent with the problem of a random walk to an absorber in three dimensions [43, 44]. In principal, these two situations are equivalent since the relative motion of the two proteins while unaligned $\mathcal{A} 1$ can be approximately understood as an isotropic random walk, and the criterion for going over to translational alignment $\mathcal{A} 2$ reflects an absorbing boundary in the configuration space of relative positions.

The distribution of resting times $T_{\text {on }}$ (plotted with the cross-symbol in Fig. 7) follows the same power law as $f\left(T_{\text {off }}\right)$, but the exponential cutoff occurs much earlier. The reason is 
that here the cutoff is determined by the region in configuration space where the two model proteins are in state $\mathcal{A} 2$. As this is much smaller than the whole volume of the boundary box, in which they are unaligned and therefore in state $\mathcal{A} 1$, a random walk in state $\mathcal{A} 2$ will end earlier.

The differences we obtain in the distributions of $T_{\text {on }}$ and $T_{\text {off }}$ when using the variants $\mathcal{M} 2$ and $\mathcal{M} 3$ compared to $\mathcal{M} 1$ are generally very small and unlikely to account for any deviations in the overall encounter rates. Also, the distribution of $N$ is always well described by a single exponential decay. However, the inverse decay length $\langle N\rangle$ significantly varies between the different situations. Therefore, changes in the overall encounter rate are mainly caused by a different probability for reaching state $\mathcal{A} 3$ from state $\mathcal{A} 2$. This is reasonable when considering that the interactions are strongly localized and can thus only act while the system is in the aligned state $\mathcal{A} 2$.

\section{Three bimolecular systems with different physico-chemical interface properties}

So far we have only considered Barnase:Barstar $(\mathcal{S} 1)$ to demonstrate how our computational model works. We now use our setup for a more comprehensive investigation. In particular, we also apply our method to two other systems, cytochrome c and its peroxidase $(\mathcal{S} 2)$ as well as the p53:MDM2 complex $(\mathcal{S} 3)$. Those represent systems with different interface characteristics and where the role of electrostatics is either much stronger $(\mathcal{S} 2)$ or much weaker $(\mathcal{S} 3)$ than for $\mathcal{S} 1$. To this end, all the previously described quantities were measured for 8 different concentrations $c=\{125,250,500,750,1250,2500,5000,7500\}$ pM. Furthermore, to find out how the choice of the radius of the reactive patch affects the results, we used patch radii of $r=6 \AA$ and $r=3 \AA$ in addition to the initially considered value of $r=10 \AA$.

Tab. II lists the encounter rates $k$ as obtained from these simulations. The rates are all roughly of the same order of magnitude. Yet several interesting qualitative features are readily apparent. First, for decreasing patch sizes, the rates generally decrease. Second, this effect is weaker for $\mathcal{M} 2$ compared to $\mathcal{M} 1$, which basically means that the electrostatic attraction and orientation due to the dipole interaction are indeed enhancing the encounter. The strongest effect of the electrostatic interaction is obtained for Cytc:CCP, which is the system with the largest monopole and dipole and the best alignment of the directions of 
the dipoles and the reactive patches. On the other hand p53:MDM2 is nearly unaffected by the effective charges, due to its weak monopole charges and, additionally, an unfavorable alignment of the dipolar interaction and the reactive surface area. Furthermore, regarding the results with detailed steric structure $\mathcal{M} 3$, the effect on the rate is correlated with the deviations of the protein forms from the spherical excluded volume approach in $\mathcal{M} 1$ and M2. This deviation is smallest for Cytc:CCP and largest for p53:MDM2.

The findings for the encounter rate $k$ are also reflected in the results for $\langle N\rangle$. As expected, an increase in $k$ correlates with a decrease in $\langle N\rangle$. The only exception is Cytc:CCP observed in $\mathcal{M}$, which is also special in regard to the effect of patch size. Here, the effective Coulombic interaction is strongest and the dipole moment is best aligned with the reactive patches. Therefore, having reached state $\mathcal{A} 2$ once, the proteins do systematically orient towards $\mathcal{A} 3$, while they are additionally strongly steered back towards $\mathcal{A} 2$ when loosing their translational alignment. This behavior is the stronger the closer the model proteins have approached once - i.e. for the case of small patch sizes, where state $\mathcal{A} 2$ implies the smallest distance. While this only explains the inversion in the $\langle N\rangle$ behavior as a function of patch size $r, k$ is obviously still slightly decreasing with smaller patch sizes. This can be explained by the fact that the time to the first approach of state $\mathcal{A} 2$ is larger for smaller patches, as this implies a smaller relative distance. This obviously compensates the fact that afterwards the encounter is formed even quicker, as reflected by the decreasing $\langle N\rangle$.

The strong correlation between the encounter rate $k$ and the mean number of contacts $\langle N\rangle$ is also evident from the correlation plot in Fig. 8. Indeed, $k \sim\langle N\rangle^{-1}$ seems valid for most of the different systems and models. It is noteworthy that the prefactor is very similar in all cases. Basically, this means that one unsuccessful contact takes the same amount of time on average, no matter what the local details of the system are. This gets more obvious recalling the distributions of the resting and return times $T_{\text {on }}$ and $T_{\text {off }}$ in Fig. 7 , which shows that $\left\langle T_{\text {off }}\right\rangle>\left\langle T_{\text {on }}\right\rangle$. As the average time for one contact will be approximately $\left\langle T_{\text {on }}\right\rangle+\left\langle T_{\text {off }}\right\rangle$, it is dominated by $T_{\text {off }}$, which is only marginally influenced by the local details of the system and the chosen model. Therefore it can be concluded, that for $\mathcal{S} 1$ and $\mathcal{S} 3$ the incorporation of a more detailed modeling approach influences $k$ and $\langle N\rangle$, but not the overall characteristics of the encounter process.

The only exceptions for the clear correlation of $k$ and $\langle N\rangle$ are $\mathcal{M} 2$ and $\mathcal{M} 3$ for the case Cytc:CCP $(\mathcal{S} 2)$, where $k$ is nearly independent of $\langle N\rangle$ because of the strong electrostatic 
interaction. This is consistent with the earlier finding, that the behavior of Cytc:CCP is qualitatively different [19], as its electrostatic interactions would facilitate long-lived nonspecific encounters between the proteins that allowed the severe orientational criteria for reaction to be overcome by rotational diffusion. For all three systems studied, in $\mathcal{M} 3$ the smallest patch size $r=3 \AA$ leads to a somewhat artificial slowing down, because in this case an overlap of the patches is rather hindered by the beads modeling the protein structure.

\section{E. Size of the reaction patches}

We next address the dependence of the data on the size of the reaction patches in more detail. This behavior is exemplary studied with the Barnase:Barstar model system. In Fig. 9, the encounter frequency has been obtained from simulations for Barnase:Barstar-like model particles in the framework of $\mathcal{M} 1$ at several concentrations $c_{0}=\{5 \mu \mathrm{M}, 125 \mathrm{nM}, 2.5 \mathrm{nM}, 125 \mathrm{pM}\}$ and varying patch sizes $r$. All values in the figure have been scaled with the concentration, which leads to data collapse. It is obvious that as $r$ gets larger than $2 R$ at around $r=40 \AA$, the reactive patch covers the whole model particle and we therefore cross over to the Smoluchowski limit of isotropic reactivity, where $k \sim r$. However, at high densities and large $r$, the patches span a large part of the simulation box of edge length $L$, and do immediately encounter for a threshold value of $r=r_{\max }=L \sqrt{3} / 4$, where the sum of the patch diameters $4 r$ equals the triagonal. Thus, the encounter frequency must diverge with $\sim 1 /\left(r_{\max }-r\right)^{\alpha}$, where we suppose $\alpha=3$, as the volume of configurational space without immediate encounter is decreasing with $r^{3}$. This assumption in addition with

the Smoluchowski behavior would lead to $k \sim r /\left(r_{\max }-r\right)^{3}$ for large $r$, which follows the data in Fig. 9 well (black dashed lines).

As already mentioned it is well known that the electrostatic interaction of proteins can severely increase the association rate. However, under physiological salt conditions, Coulombic interactions are screened by counter ions in the solution on a small length scale of approximately $1 \mathrm{~nm}$. Thus, deviations from case $\mathcal{M} 1$ without effective charges will only arise for small $r$. Fig. 10 shows the results of respective simulations for $\mathcal{M} 2$ compared to the results for $\mathcal{M} 1$, as considered before. Indeed, for large patch radii $r$, the results are similar, while for smaller $r$, the encounter rates in $\mathcal{M} 2$ are clearly higher compared to $\mathcal{M} 1$. However, the crossover to a power law behavior with roughly $\sim r^{9 / 4}$ can be detected for very small $r$, 
but at a prefactor of about 50 times larger than for $\mathcal{M} 1$.

\section{DISCUSSION}

The main goal of this work was to model protein encounter in a generic framework which allows us to include molecular details without making future upscaling to larger complexes impossible. Our model approach incorporates steric, electrostatic and thermal interactions of the proteins considered. These interactions are thought to be the major factors governing protein encounter. Not included are conformational changes of the proteins upon association, related entropic terms, and the molecular nature of the surrounding solvent that becomes relevant at close distances. The model parameters are extracted from the atomic structures available in the protein data bank by generally applicable protocols as described in Sect. II. In principle, these methods of data extraction can be fully automatized.

The biggest advantage of our coarse-grained model is the possibility to extend the simulations to large scales in terms of particle numbers, time and system size. In many of the earlier studies [39, 45, 46, 47], the system was prepared already close to encounter and the overall association rate was then calculated via a sophisticated path-integral like procedure. In contrast, our simulations account for the whole process of diffusional encounter and is thus rather general, allowing for spanning large time scales via our adaptive time step algorithm. In particular, each set of simulations consists of $10^{4}$ to $10^{5}$ runs of lengths up to the order of seconds and could be performed on a standard CPU within hours of computer time.

Being able to directly obtain the first passage times (FPT) of the encounter processes in our model allows to check the validity of several phenomenological assumptions. First of all, the FPT distribution matched very well a Poisson process with a single stochastic rate, as seen in Fig. 2, which validates the notions of encounter and association. Moreover, our approach provides two ways of controlling the particle density and for both cases the results corresponded well to the expected scaling. First, the concentration is inversely correlated with the size of the periodic boundary simulation box. We show that the encounter rate grows linearly with the particle concentration. Furthermore, leaving the box size constant, the concentration can also be varied by adding a higher number $N$ of particles. Considering

only the first encounter of any of the possible complementary pairs of model particles, the 
mean first passage time to this event is not only lowered by a factor $N$ but we show that the expected behavior is an enhancement of the encounter frequency by $N^{2}$, which is nicely matched by the results of the simulations. Therefore we can conclude that the computational model studied here satisfies the general requirement of stochastic bimolecular association processes that describe binding by a single rate constant.

To test our model against known results we have chosen three well-known bimolecular systems with different characteristics. The Barnase:Barstar complex is the gold standard for protein-protein association and characterized by relatively strong electrostatic steering. The association of Cytochrome c and its peroxidase is even more strongly affected by Coulombic attraction. Here, both proteins have a rather spherical form. Finally, the p53:MDM2 complex has a different characteristic with a very small net charge and a deep cleft perfectly matching the small peptide p53, whose reactive area is therefore nearly spanning over its whole surface. These model systems were purposely chosen to check whether our effective representations of the protein properties would lead to reasonable and significantly distinguishable results. Indeed, this is the case as the discussion of the results in Tab. II in the respective section shows.

When comparing the results for the encounter rates in Tab. II with previous studies from the field of bimolecular protein association, several aspects have to be kept in mind. First, throughout this study, we do only consider the encounter of our model particles. As explained in the beginning, the complete association of the complex still lacks the step over a final free energy barrier, which is due to effects such as the dehydration of the protein surfaces and thus requires more detailed modelling. In the framework of our approach, this final step could be modelled by a stochastic rate criterion, where the rate can be obtained by transition state theory from the energy landscapes characterized in atomistic calculations. In any case, any additional process to be included can only lower the values found in our study.

In the work on Barnase:Barstar by Schreiber et al. [5], the authors reported that the association between Barnase and Barstar is a diffusion-limited reaction. The argument for this is that the association rates at high ionic concentrations in the solution, i.e. for the limit in which the electrostatic steering gets negligible, are clearly lowered by the addition of glycerol, which will lead to slower diffusion. Assuming diffusion control, the reactive step over the final barrier should be kinetically unimportant, as generally discussed in Ref. 
[48]. Indeed, we see that our results for the encounter rates lead to values in the correct order of magnitude of $k \approx 10^{9} \mathrm{M}^{-1} \mathrm{~s}^{-1}$, which is similar to the experimental value obtained by Schreiber et al. for the association constant of Barnase:Barstar $k=8 \cdot 10^{8} \mathrm{M}^{-1} \mathrm{~s}^{-1}$ at physiological salt [49]. However, the basal association rate, i.e. the rate at high ionic strength, is reported as $k<10^{6} \mathrm{M}^{-1} \mathrm{~s}^{-1}$ from experiments [5]. Given that the association process of Brn:Brs is diffusion limited, these findings should actually coincide with our values for $\mathcal{M} 1$. But as we already discussed in the results section, in our simulations the influence of the effective electrostatics introduced in $\mathcal{M} 2$ do not result in such a drastic change of the encounter behavior.

In several earlier approaches, similar problems have been addressed by computational and analytical studies. In work by Zhou and coworkers, basal encounter rates for particles with reactive patches have been found to be $k=4 \cdot 10^{6} \mathrm{M}^{-1} \mathrm{~s}^{-1}[15]$ and $k=10^{7} \mathrm{M}^{-1} \mathrm{~s}^{-1}[20$, that is closer to the basal rates reported by Schreiber and coworkers. It has to be noted that, in both cases, the patches were flat areas above the surface of the spherical model particles, which had a smaller angular extension compared to our cases, and especially required a much closer translational approach $(0.7 \AA$ in [15]) to form the encounter. If we expand the graph in Fig. 9 to smaller patch radii like $r=1 \AA$, we also find basal rates in the order of $k=10^{7} \mathrm{M}^{-1} \mathrm{~s}^{-1}$. Also, the deviation between $\mathcal{M} 1$ and $\mathcal{M} 2$, i.e. the influence of the effective electrostatics, is more prominent and could enhance the encounter rate by about two orders of magnitude, which is consistent with the findings in the previously cited work. There, the effect of Coulombic interaction is reflected with a Boltzmann factor due to a pairwise Coulomb energy. This approach works well, as shown in Ref. [47], and has been recently used in a more complex model study of the energy landscape of protein-protein association [50, 51].

Any model for the reaction patches has to rely on results obtained from more detailed modeling. The surface of a protein is typically densely covered by water molecules due to the hydrophilic nature of its surface. This hydration shell has a thickness of about $3 \AA$ and will therefore in principal hinder the approach of two proteins to distances below $6 \AA$. Setting the encounter patches to values below this threshold of $3 \AA$ would then mean that part of the dehydration would already have happened before the encounter is actually formed, which is probably hardly described by simple diffusion with drift. Moreover, all of the considered protein systems feature distinct key-lock binding interfaces regarding the steric structure, 
apart from some flexibility due to intrinsic thermal motion. Therefore, it makes sense to represent the encounter area by a three-dimensional extended object rather than by a flat surface region. Indeed, the results of our studies show that our approach is capable of reproducing encounter rates in a reasonable order of magnitude, qualitatively reproducing generally expected features. In an in-depth investigation of the dependency of the encounter rate and the patch radius it is shown, that the choice of the geometry of the reactive area is at least as crucial for the results as definition of the model interactions and its parameters. In principal, one could think of the patch radius as a valuable tuning parameter to fit experimental results and the encounter kinetics in the computational model.

Our approach makes it possible to observe general features of the encounter process. In particular, we dissect the pathway to the encounter complex in several levels of alignment between our model proteins. As we observe the full trajectory to encounter in our simulations, we are able to extract the number of unsuccessful contacts $N$ between the proteins until they finally reach a reasonably aligned state to bind. The distribution of $N$ is again in all cases well described by a single exponential decay. This behavior is not obvious as the probability of success for one contact is depending on several aspects of diffusion in a complex manner. First, the closer the rotational alignment at the beginning of the contact is to the encounter state, the higher is the probability of success. Second, this initial alignment is also coupled to the last contact if the time in between $T_{\text {off }}$ is small. Finally, longer contact resting times $T_{\text {on }}$ also increase the probability of encounter. It is interesting, that all these effects still lead to a simple Poisson distribution of the number of contacts $N$ when averaging over the initial conditions as it is done in this work. Furthermore, we find that the distributions of these resting and return times cannot be described by a Poisson process, but are consistent with the expectations for a spatially constricted random walk in three dimensions. We find that the particular mean FPT to encounter is in most of the cases directly proportional to the number of unsuccessful contacts. This seems to be a very fundamental qualitative feature irrespective of the details of the proteins and the applied model. However, for Cytc:CCP the behavior is qualitatively different, which is consistent with earlier studies of this highly electrostatically steered complex.

In summary, here we have presented a Langevin equation approach to protein-protein association which in principle allows us to combine long simulation times and large systems with molecular details of the involved proteins. This first study has focused on bimolecular 
reactions and has proven that this approach is capable of reproducing known association rates with a reasonable dependance on the main parameters involved. One special strength of our approach is that it allows us to address the details of the binding pathway, for example by measuring the statistics of unsuccessful contacts before encounter. In the future, this approach will be extended to large protein complexes of special biological interest.

\section{Acknowledgments}

We thank Christian Korn for many helpful discussions. This work was supported by the Volkswagen Foundation through grants I/80469 and I/80470 to V.H. and U.S.S., respectively.

J.S. and U.S.S. are supported by the Center for Modelling and Simulation in the Biosciences (BIOMS) at Heidelberg and by the Karlsruhe Institute of Technology (KIT) through its Concept for the Future.

[1] V. Helms, Principles of Computational Cell Biology: From Protein Complexes to Cellular Networks (Wiley-Vch, 2008), 1st ed.

[2] B. Alberts, D. Bray, and J. Lewis, Molecular Biology of the Cell (Taylor \& Francis, New York, 2002), 4th ed.

[3] A.-C. Gavin and et al., Nature 440, 631 (2006).

[4] U. de Lichtenberg, L. J. Jensen, S. Brunak, and P. Bork, Science 307, 724 (2005).

[5] G. Schreiber and A. R. Fersht, Nat. Struct. Biol. 3, 427 (1996).

[6] G. Schreiber, Curr. Op. Struct. Bio. 12, 41 (2002).

[7] A. Spaar, C. Dammer, R. R. Gabdoulline, R. C. Wade, and V. Helms, Biophys. J. 90, 1913 (2006).

[8] O. G. Berg and P. H. van Hippel, Annu. Rev. Biophys. Biophy. Chem. 14, 131 (1985).

[9] M. Ahmad, W. Gu, and V. Helms, Angewandte Chemie (2008).

[10] O. F. Lange, N.-A. Lakomek, C. Fares, G. F. Schroder, K. F. A. Walter, S. Becker, J. Meiler, H. Grubmuller, C. Griesinger, and B. L. de Groot, Science 320, 1471 (2008).

[11] M. Smoluchowski, Z. Phys. Chem. 92, 129 (1917).

[12] P. J. W. Debye, Trans. Electrochem. Soc. 82, 265 (1942). 
[13] H. C. Berg and E. M. Purcell, Biophys. J. 20, 193 (1977).

[14] D. Shoup, G. Lipari, and A. Szabo, Biophys. J. 36, 697 (1981).

[15] H. X. Zhou, Biophys. J. 64, 1711 (1993).

[16] M. Schlosshauer and D. Baker, J. Phys. Chem. B 106, 12079 (2002), ISSN 1520-6106.

[17] M. Schlosshauer and D. Baker, Protein Sci. 13, 1660 (2004).

[18] A. Szabo, K. Schulten, and Z. Schulten, J. Chem. Phys. 72, 4350 (1980).

[19] S. Northrup, J. Boles, and J. Reynolds, Science 241, 67 (1988).

[20] H. X. Zhou, Biophys. J. 73, 2441 (1997).

[21] R. Wade, R. Gabdoulline, S. Lüdemann, and V. Lounnas, Proc. Natl. Acad. Sci. USA 95, $5942(1998)$.

[22] A. Elcock, R. Gabdoulline, R. Wade, and J. McCammon, J. Mol. Biol. 291, 149 (1999).

[23] R. R. Gabdoulline and R. C. Wade, Biophys. J. 72, 1917 (1997).

[24] D. Bicout and M. Field, J. Phys. Chem. 100, 2489 (1996).

[25] S. McGuffee and A. Elcock, J. Am. Chem. Soc. 128, 12098 (2006), ISSN 0002-7863.

[26] L. Chong, S. Dempster, Z. Hendsch, L. Lee, and B. Tidor, Protein Sci. 7, 206 (1998).

[27] T. Selzer and G. Schreiber, J. Mol. Biol. 287, 409 (1999).

[28] F. Sheinerman and B. Honig, J. Mol. Biol. 318, 161 (2002).

[29] F. Dong, M. Vijayakumar, and H. Zhou, Biophys. J. 85, 49 (2003).

[30] T. Wang, S. Tomic, R. Gabdoulline, and R. Wade, Biophys. J. 87, 1618 (2004).

[31] H. Pelletier and J. Kraut, Science 258, 1748 (1992).

[32] J. G. de la Torre, M. L. Huertas, and B. Carrasco, Biophys. J. 78, 719 (2000).

[33] J. Antosiewicz, J. Briggs, and J. McCammon, Eur. Biophys. J. 24, 137 (1996).

[34] C. B. Korn and U. S. Schwarz, Phys. Rev. Lett. 97, 138103 (2006).

[35] C. B. Korn and U. S. Schwarz, J. Chem. Phys. 126, 095103 (2007).

[36] S. Ramanathan and D. C. Morse, J. Chem. Phys. 126, 094906 (2007).

[37] Y. Han, A. M. Alsayed, M. Nobili, J. Zhang, T. C. Lubensky, and A. G. Yodh, Science 314, $626(2006)$.

[38] J. S. van Zon and P. R. ten Wolde, Phys. Rev. Lett. 94, 128103 (2005).

[39] L. D. Eltis, R. G. Herbert, P. D. Baker, A. G. Mauk, and S. H. Northrup, Biochemistry 30, 3663 (1991).

[40] C. Gorba, T. Geyer, and V. Helms, J. Chem. Phys. 121, 457 (2004). 
[41] O. Byron, Biophys. J. 72, 408 (1997).

[42] N. Rai, M. Nöllmann, B. Spotorno, G. Tassara, O. Byron, and M. Rocco, Structure 13, 723 (2005).

[43] G. Pólya, Mathematische Annalen 84, 149 (1921).

[44] B. D. Hughes, Random walks and random environments (Clarendon Press, Oxford, 1995).

[45] S. H. Northrup, S. A. Allison, and J. A. McCammon, J. Chem. Phys. 80, 1517 (1984).

[46] S. Northrup and H. Erickson, Proc. Natl. Acad. Sci. USA 89, 3338 (1992).

[47] H. X. Zhou and A. Szabo, Biophys. J. 71, 2440 (1996).

[48] G. I. Bell, Science 200, 618 (1978).

[49] G. Schreiber and A. R. Fersht, Biochemistry 32, 5145 (1993).

[50] R. Alsallaq and H.-X. Zhou, Structure 15, 215 (2007).

[51] R. Alsallaq and H.-X. Zhou, Biophys. J. 92, 1486 (2007).

[52] A. M. Buckle, G. Schreiber, and A. R. Fersht, Biochemistry 33, 8878 (1994), ISSN 0006-2960.

[53] P. H. Kussie, S. Gorina, V. Marechal, B. Elenbaas, J. Moreau, A. J. Levine, and N. P. Pavletich, Science 274, 948 (1996). 
TABLE I: Protein structures and parameters used in the study. The coordinates of the patches $\left(\mathbf{r}_{\text {patch }}\right)$ and the dipole moment $(\mathbf{p})$ are given relative to the center of mass.

\begin{tabular}{|c|c|c|c|c|c|c|c|}
\hline Protein & System & PDB code & Ref. & $R_{\mathrm{gyr}} / \AA$ & $q / e$ & $\mathbf{r}_{\text {patch }} / \AA$ & $\mathbf{p} / \AA e$ \\
\hline Barnase & $\mathcal{S} 1$ & $1 \mathrm{brs}$ & {$[23,52]$} & 14.68 & 2 & $(5.43,-4.75,-3.41)$ & $(3.84,-0.67,-36.15)$ \\
\hline Barstar & & & & 13.42 & -6 & $(-6.05,3.75,6.34)$ & $(54.75,-14.39,0.621)$ \\
\hline Cytc & $\mathcal{S} 2$ & $2 \mathrm{PCC}$ & 31 & 13.89 & 6 & $(-2.08,7.99,-3.83)$ & $(-7.03,117.79,-10.99)$ \\
\hline $\mathrm{CCP}$ & & & & 20.00 & -13 & $(8.89,-8.19,11.46)$ & $(-23.47,93.85,-171.82)$ \\
\hline p53 & $\mathcal{S} 3$ & $1 Y C R$ & 53 & 10.20 & -2 & $(0.28,0.21,0.59)$ & $(21.64,-17.73,-17.12)$ \\
\hline MDM2 & & & & 16.81 & 1 & $(1.577,-4.74,-0.51)$ & $(75.07,14.51,4.67)$ \\
\hline
\end{tabular}


TABLE II: Encounter rates $k$ which have been averaged over several simulations at different concentrations as given in the text. The values are given in $k / 10^{9} \mathrm{M}^{-1} \mathrm{~s}^{-1}$ for the three different versions of our model. $\langle N\rangle$ are average values for the number of unsuccessful contacts before encounter. $\langle N\rangle$ is basically independent of the concentration. Therefore it is again averaged over the different simulations for each of the chosen systems. The errors were determined by one standard deviation from the 8 values obtained at different concentrations. Some of the choices for the patch radius were not applicable to $\mathcal{M} 3$, as for these cases an encounter was completely prevented by the detailed excluded volume model.

\begin{tabular}{|c|c|c|c|c|c|c|c|}
\hline System & Patch radius & $k(\mathcal{M} 1)$ & $k(\mathcal{M} 2)$ & $k(\mathcal{M} 3)$ & $\langle N\rangle(\mathcal{M} 1)$ & $\langle N\rangle(\mathcal{M} 2)$ & $\langle N\rangle(\mathcal{M} 3)$ \\
\hline \multirow[t]{3}{*}{ Brn:Brs } & 10.0 & $1.56 \pm 0.04$ & $2.76 \pm 0.07$ & $2.02 \pm 0.02$ & $474 \pm 2$ & $198 \pm 5$ & $282 \pm 10$ \\
\hline & 6.0 & $0.57 \pm 0.01$ & $2.13 \pm 0.01$ & $1.34 \pm 0.08$ & $1140 \pm 4$ & $232 \pm 8$ & $534 \pm 50$ \\
\hline & 3.0 & $0.13 \pm 0.001$ & $1.28 \pm 0.03$ & - & $4120 \pm 10$ & $653 \pm 15$ & - \\
\hline \multirow[t]{3}{*}{ Cytc:CCP } & 10.0 & $1.12 \pm 0.02$ & $4.31 \pm 0.20$ & $4.15 \pm 0.15$ & $842 \pm 3$ & $61 \pm 5$ & $71 \pm 7$ \\
\hline & 6.0 & $0.40 \pm 0.01$ & $4.29 \pm 0.21$ & $4.05 \pm 0.09$ & $2040 \pm 20$ & $30 \pm 3$ & $77 \pm 10$ \\
\hline & 3.0 & $0.09 \pm 0.001$ & $4.03 \pm 0.05$ & $0.21 \pm 0.02$ & $7540 \pm 63$ & $21 \pm 3$ & $4160 \pm 375$ \\
\hline \multirow[t]{3}{*}{ p53:MDM2 } & 10.0 & $2.05 \pm 0.05$ & $2.51 \pm 0.04$ & $1.27 \pm 0.02$ & $362 \pm 2$ & $266 \pm 4$ & $823 \pm 10$ \\
\hline & 6.0 & $0.80 \pm 0.01$ & $1.12 \pm 0.01$ & $0.15 \pm 0.01$ & $815 \pm 10$ & $582 \pm 13$ & $8720 \pm 200$ \\
\hline & 3.0 & $0.19 \pm 0.002$ & $0.28 \pm 0.01$ & - & $2900 \pm 35$ & $2550 \pm 30$ & - \\
\hline
\end{tabular}


FIG. 1: Scheme to visualize the different variants of the model for the three considered model systems. The color code is: yellow for Barnase, cytochrome c and p53; green for Barstar, cytochrome c peroxidase and MDM2. The respective reaction patches are shown in white. $\mathcal{M} 1$ only includes a simple steric interaction. $\mathcal{M} 2$ has an additional effective electrostatic interaction, here denoted with red arrows showing the direction of the dipole of the model particles. In $\mathcal{M} 3$, the excluded volume is modeled in more detail as a collection of smaller beads. The transparent blue spherical surface marks the volume used in $\mathcal{M} 1$ and $\mathcal{M} 2$ for the sake of comparison. Finally, the bottom row shows surface representations of the atomistic structures taken from the protein database.

FIG. 2: Logarithmic plot of the distribution of the first passage time to encounter $T$ between a single pair of Barnase and Barstar model particles in a cubic boundary box of edge length $L=2370 \AA$, representing a concentration of $0.125 \mu \mathrm{M}$ for each protein. The dashed line represents a single exponential fit to the data points, which shows the expected behavior with respect to the encounter frequency $k=\langle T\rangle^{-1}$.

FIG. 3: Simulated encounter frequencies for a single pair of Barnase and Barstar model particles in cubic boundary boxes of different sizes representing different concentrations. The dashed line is a linear fit to the data.

FIG. 4: Encounter frequency for different number of Barnase:Barstar pairs leaving the size of the boundary box constant. The red data points show the encounter frequencies as obtained from simulations, while the green line represents the function $C N^{2}$, where $N$ is the number of particle pairs and $C$ is a fitted prefactor. 
FIG. 5: Different alignment states during the encounter process. $\mathcal{A} 1$ proteins are completely unaligned. In state $\mathcal{A} 2$, referred to as contact in this paper, the proteins are translationally aligned, i.e. they are close enough to actually encounter (denoted by the overlap of the lightened area around the model particles), but lack the correct orientation. $\mathcal{A} 3$ proteins reached the encounter meaning that the reactive patches are in translational and rotational alignment.

FIG. 6: Logarithmically plotted distribution of the number of approaches $N$ between a Barnase and a Barstar particle with incorrect rotational alignment. The dashed line is an exponential fit to the data.

FIG. 7: Double-logarithmic plot of the distribution of resting and return times of the translationally aligned state ( $\mathcal{A} 2$ in Fig. 5 ).

FIG. 8: Correlation plot of encounter rate $k$ and mean number of contacts $\langle N\rangle$ with all the data from Tab. III.

FIG. 9: Encounter rates in dependency of the patch size for the Barnase:Barstar model system in the $\mathcal{M} 1$ variant.

FIG. 10: Comparison of $\mathcal{M} 1$ and $\mathcal{M} 2$ similar to Fig. 9 for small patch sizes. For larger patch sizes there is no substantial difference. 


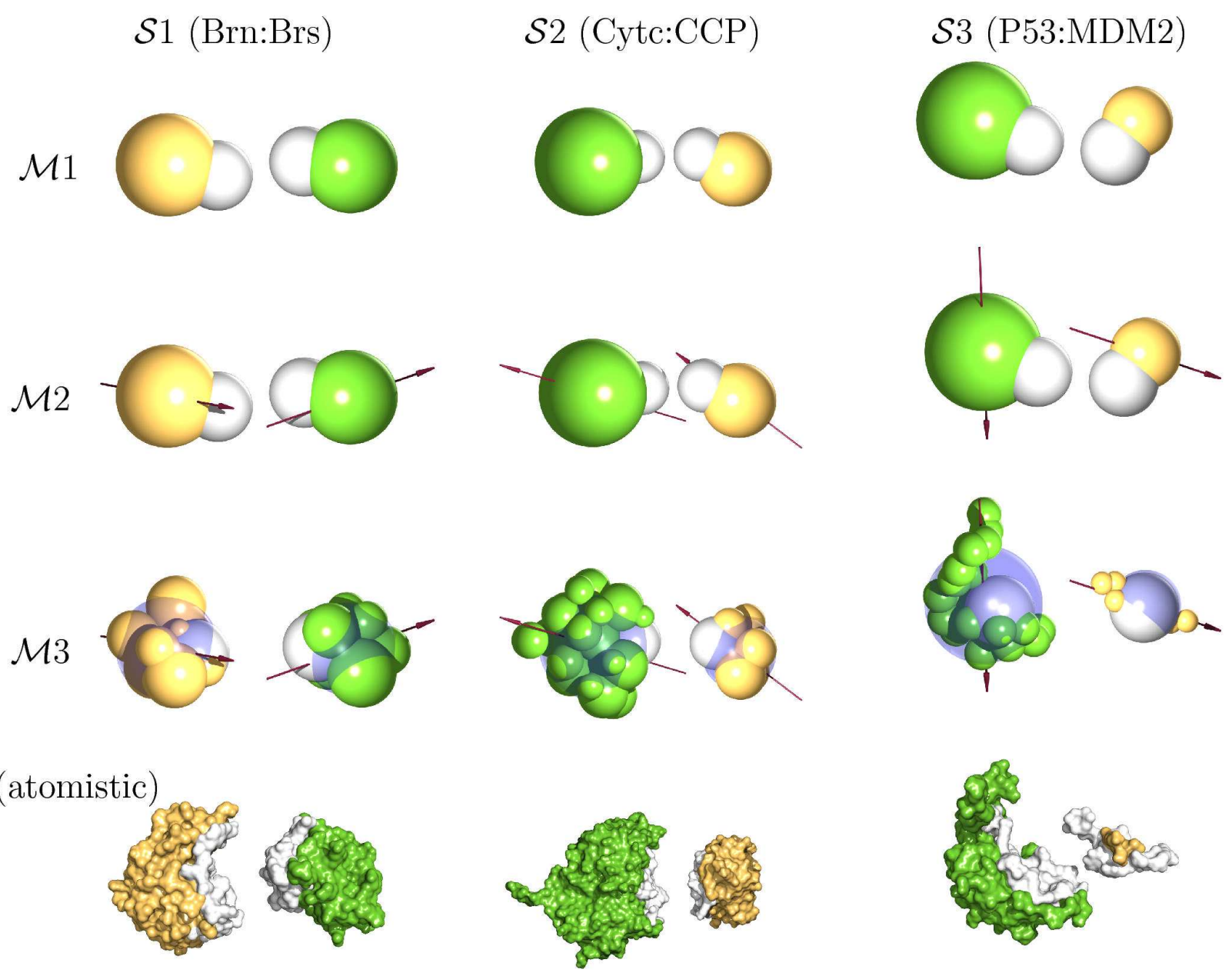

Fig. 1 


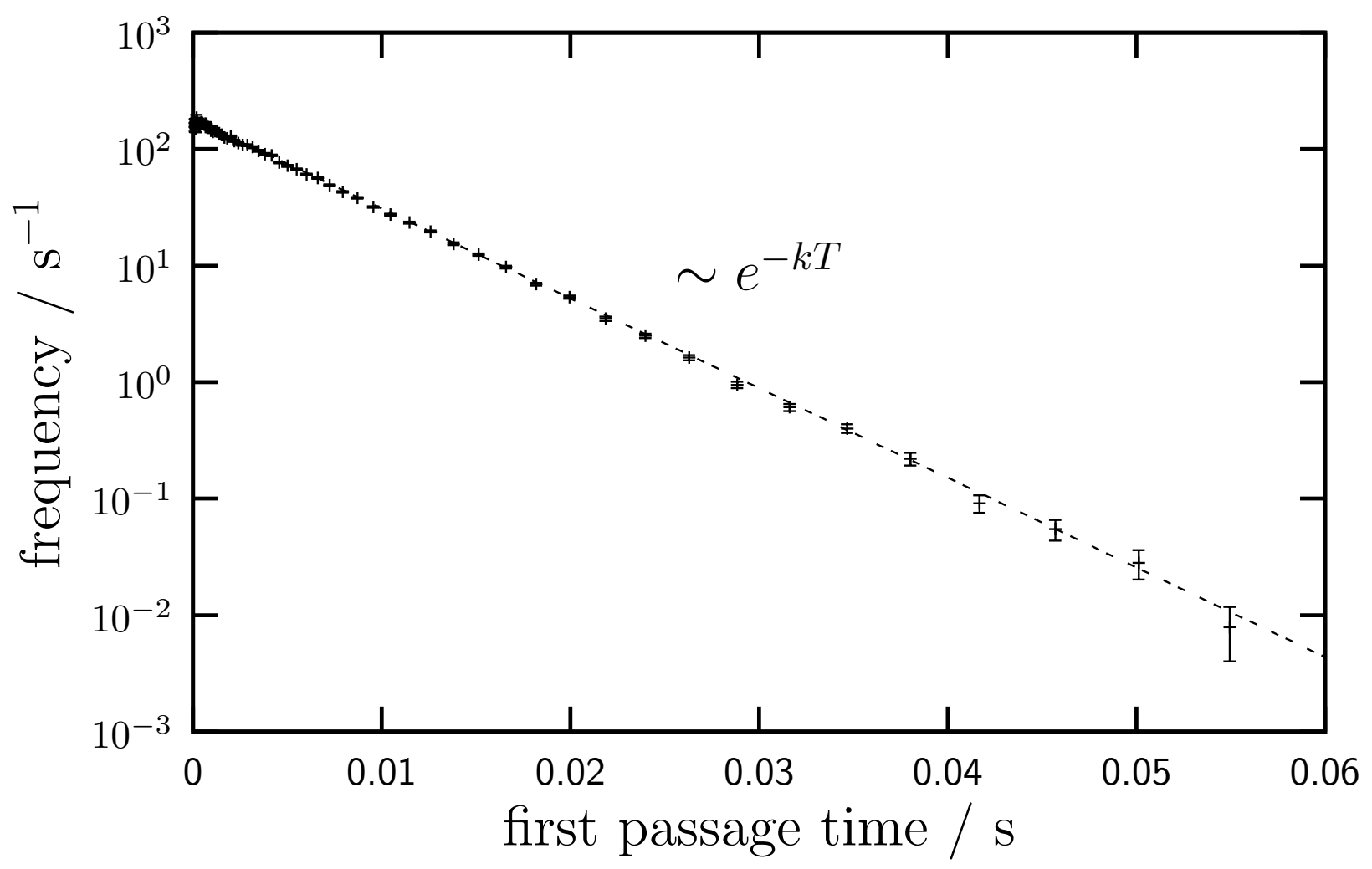

Fig. 2 


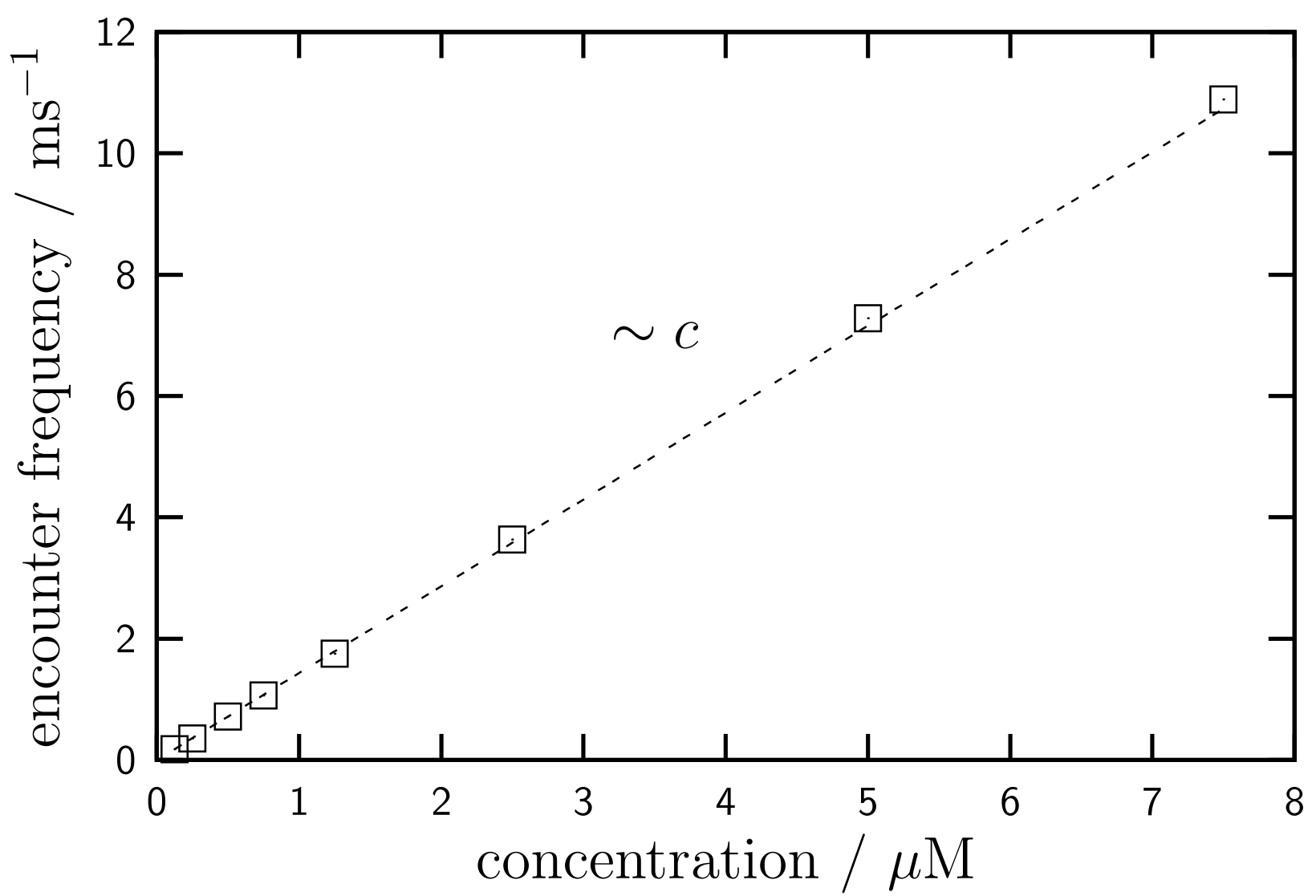

Fig. 3 


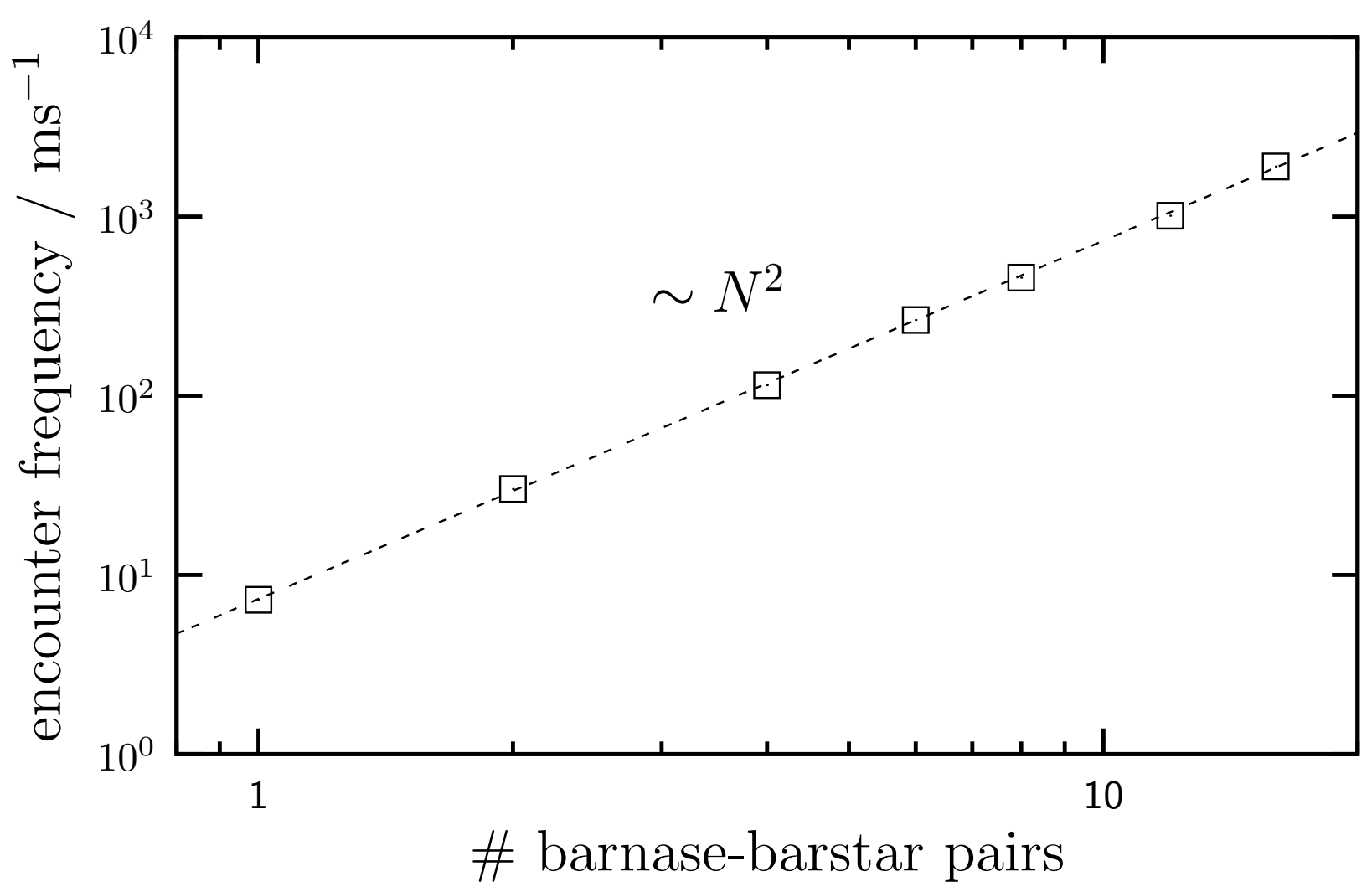

Fig. 4 

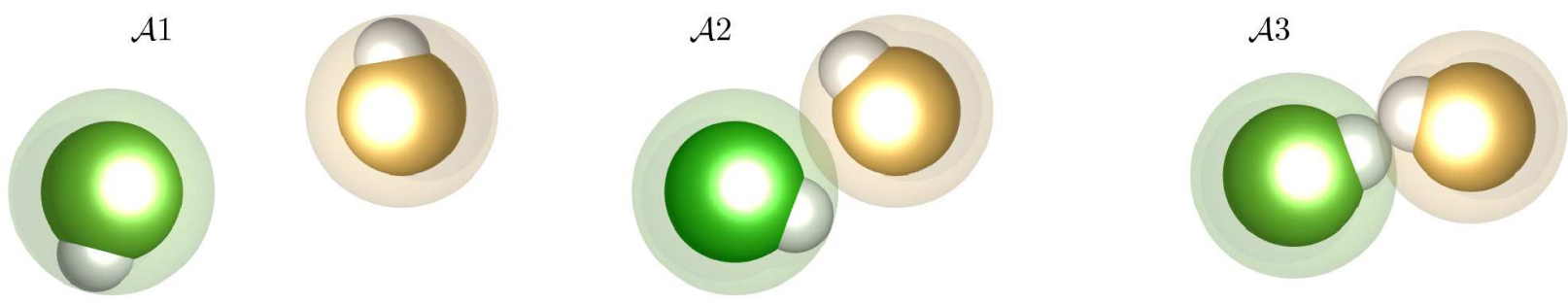

Fig. 5 


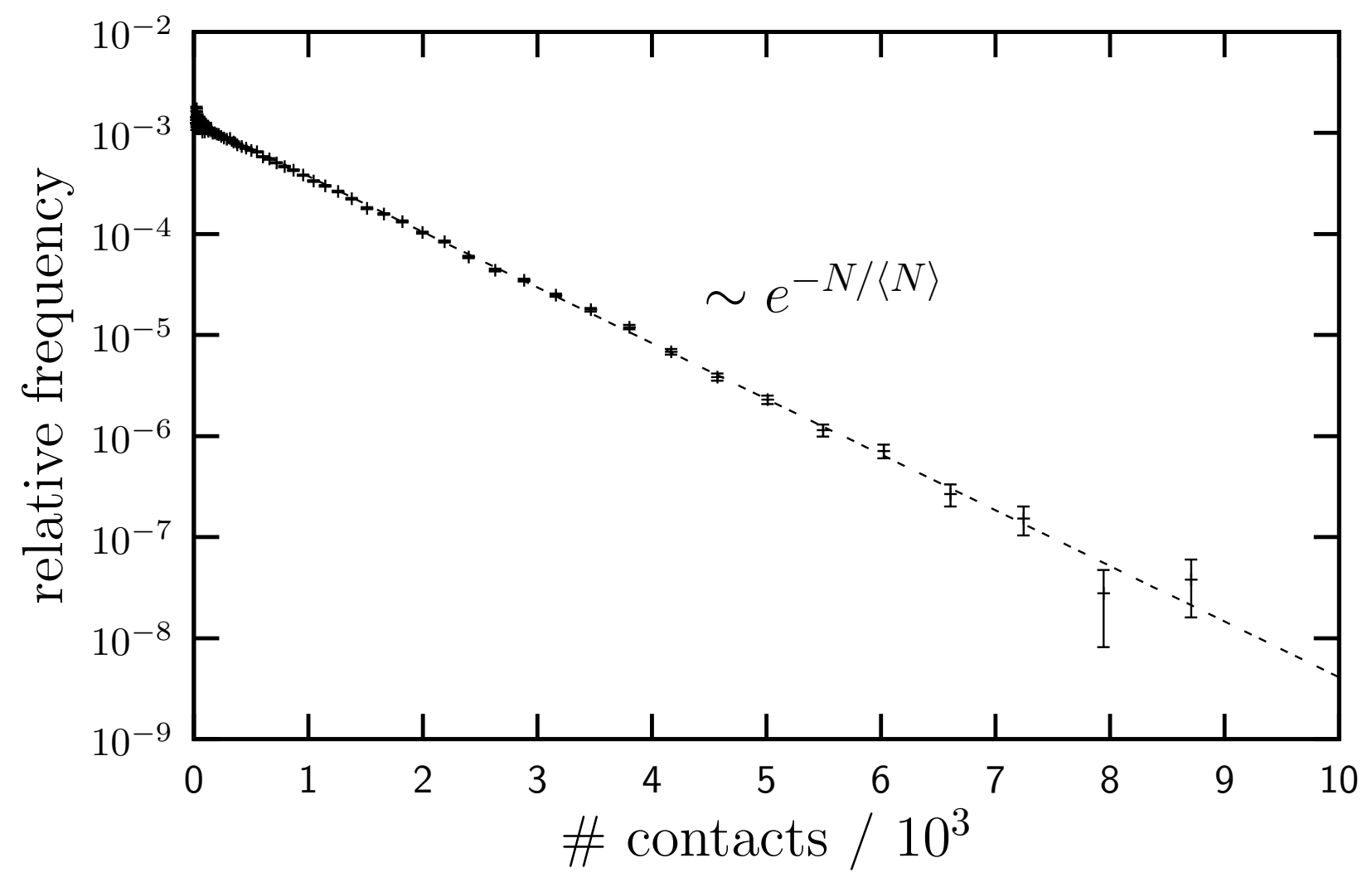

Fig. 6 


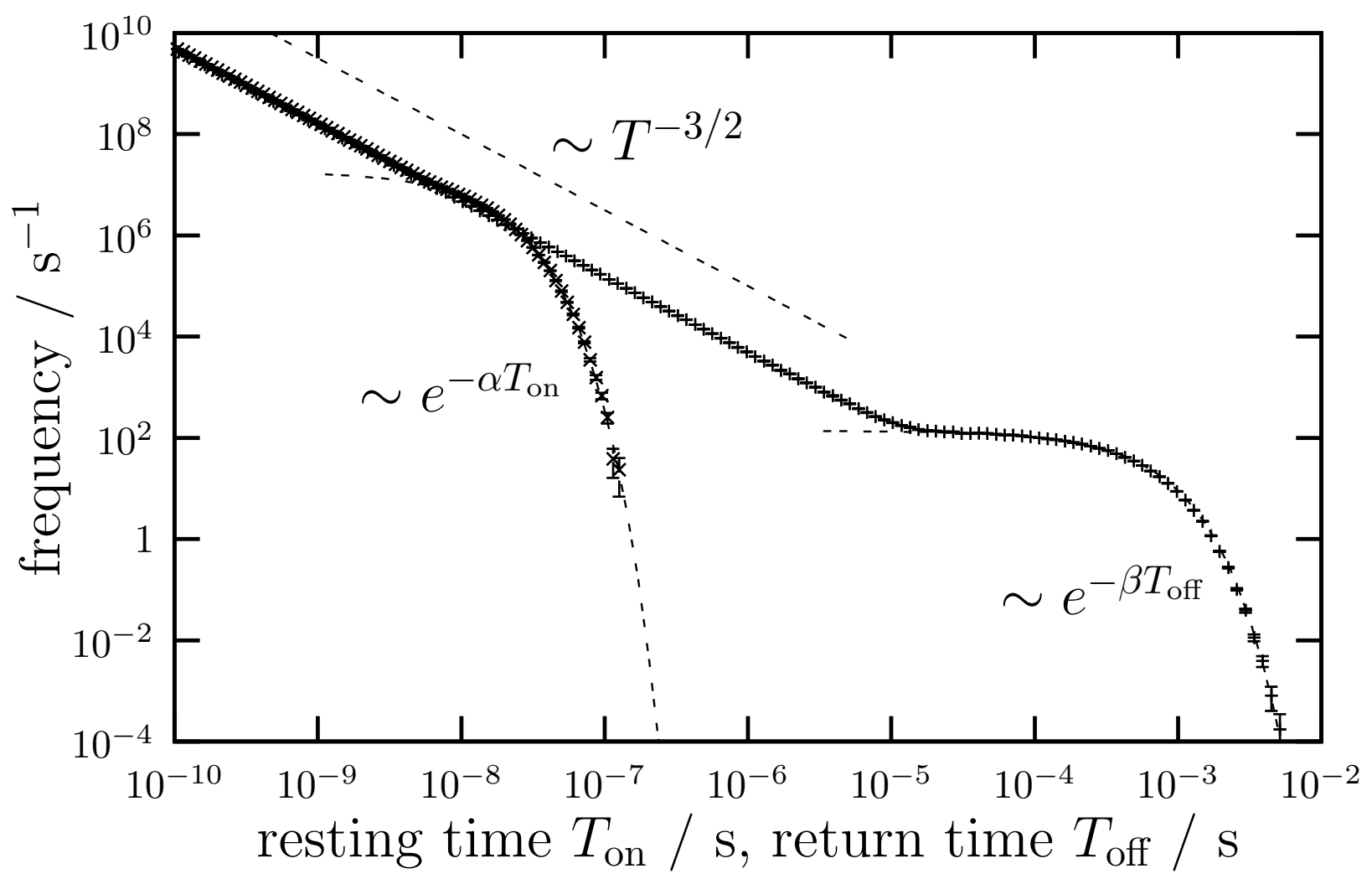

Fig. 7 


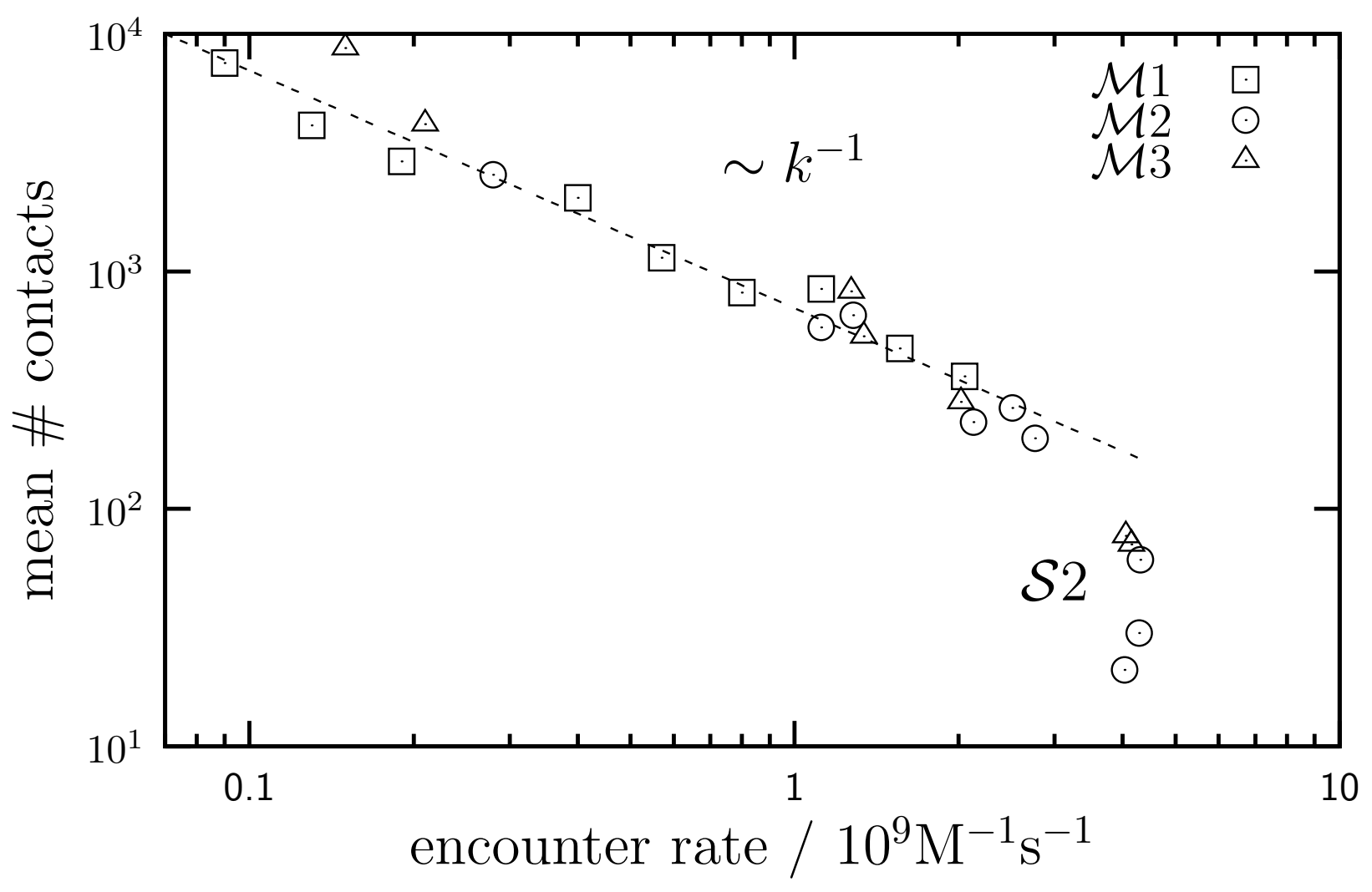

Fig. 8 


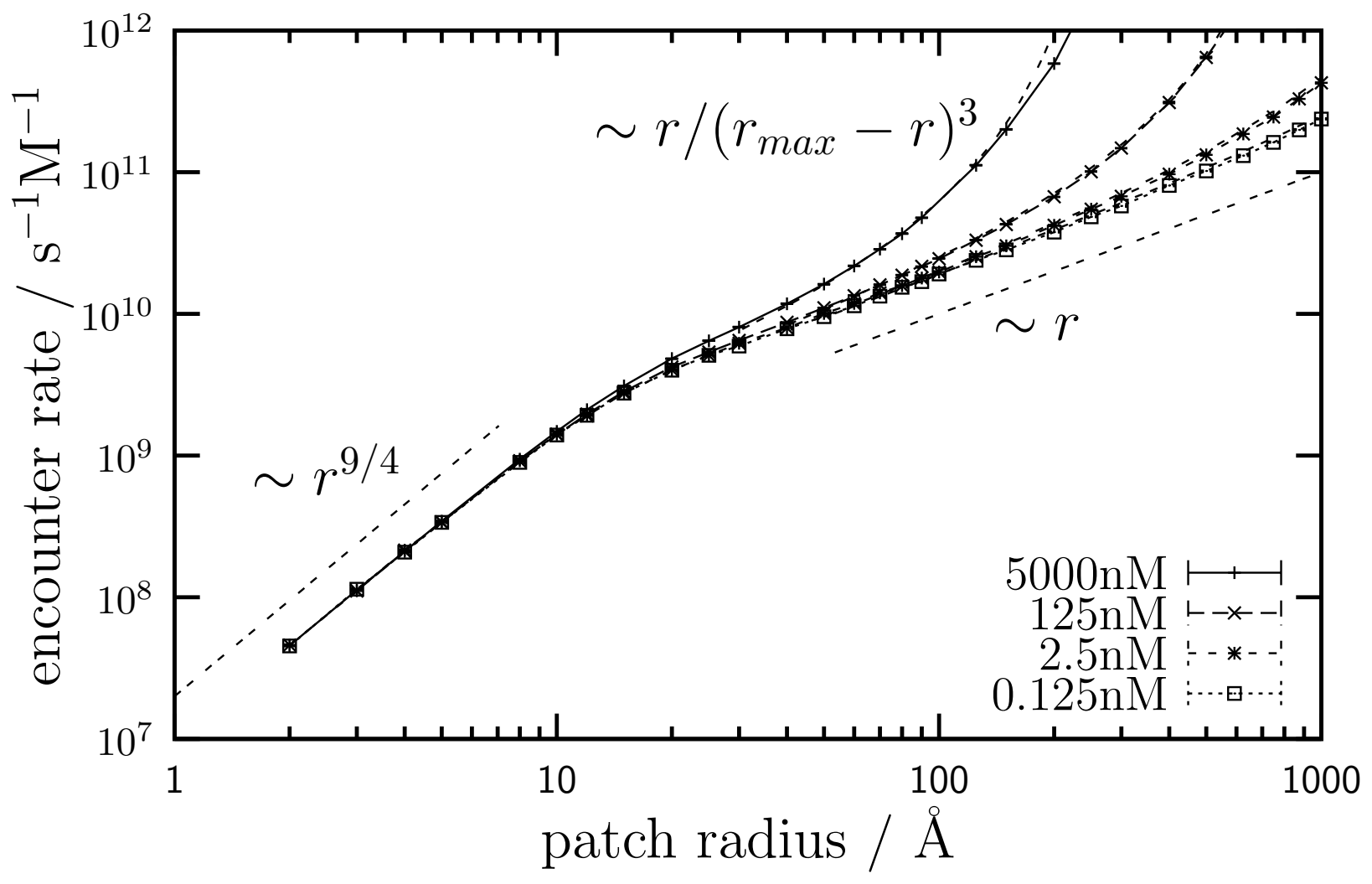

Fig. 9 


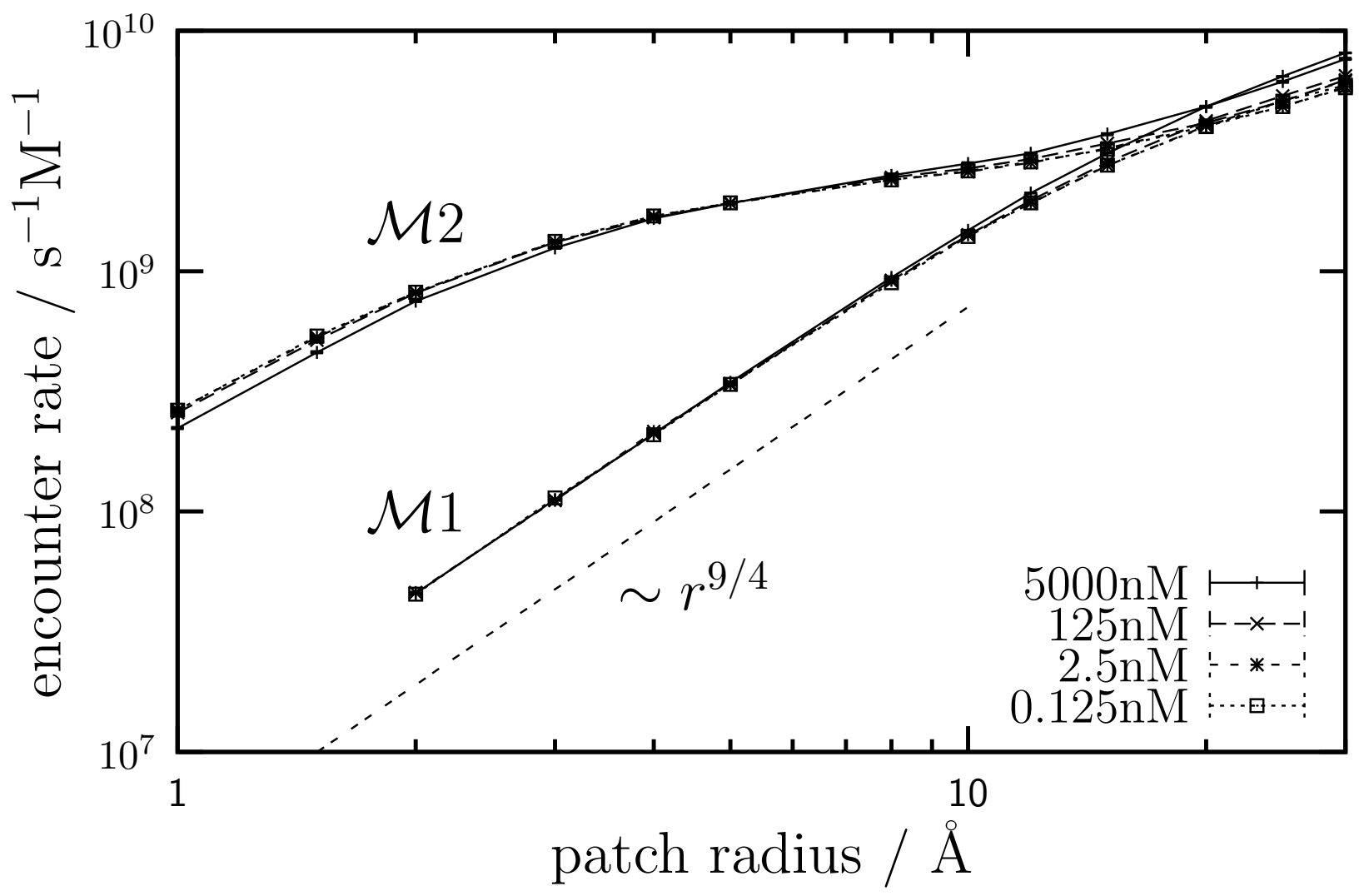

Fig. 10 